\title{
Multiplicative Degree-Kirchhoff Index of Random Polyphenyl Chains
}

\author{
Meilian Li,${ }^{1 *}$ Jinshan Xie, ${ }^{1}$ Dezhong Lian, ${ }^{1}$ and Cheng-Fu Yang ${ }^{2,3 * *}$ \\ ${ }^{1}$ School of Mathematics and Information Engineering, Longyan University, Longyan, Fujian 364012, China \\ ${ }^{2}$ Department of Chemical and Materials Engineering, National University of Kaohsiung, Kaohsiung 811, Taiwan \\ ${ }^{3}$ Department of Aeronautical Engineering, Chaoyang University of Technology, Taichung 413, Taiwan
}

(Received March 23, 2021; accepted May 31, 2021)

Keywords: multiplicative degree-Kirchhoff index, random polyphenyl chain, molecular graph

The multiplicative degree-Kirchhoff index of a connected graph is defined as the sum of the product of the resistance distances between all pairs of points and the degrees of corresponding point pairs in the graph. In this paper, we propose an exact formula to compute the expected value of the multiplicative degree-Kirchhoff index of a random polyphenyl chain. Furthermore its asymptotic property is also considered.

\section{Introduction}

Polyphenyls and their derivatives, which are used in heat exchangers, drug synthesis, organic synthesis, and so on, have attracted the attention of researchers in various fields for many years. Spiro-based compounds are a very important class of cycloalkanes in organic chemistry. The function of a spiro union is to form a linkage between two rings in spiro compounds, which consists of a single atom common to both rings. A free spiro union forms a linkage, which is the only direct union between the rings. In the past, when chemists wanted to determine the relationship between the properties of a compound and its molecular structure, it was necessary to perform many experiments and obtain a large amount of data. ${ }^{(1)}$ By using the spiro union compounds, materials, drugs, crystals, and compounds can be represented by graph structures called molecular graphs. Also, conducting polymers, such as polythiophene (PTh), polyaniline (Pani), polypyrrole (PPy), and their derivatives, have been used as the active layers of different gas sensors since the early $1980 \mathrm{~s}^{(2)}$ The thermal and chemical stabilities of conducting polymers under ambient conditions have enhanced their utilization as active sensing layers deposited by either an in situ electrochemical reaction or a chemical reaction on an electrode or an electrode array. Therefore, conducting polymers can be used to fabricate gas sensor devices, which respond to and/or detect particular toxic gases and volatile organic compounds (VOCs). These devices are also used as ion-trapping devices at ambient temperature for quality control in production and for environmental remediation. ${ }^{(2,3)}$ When conducting polymers are used in different sensor devices, their resistance is an important factor affecting their sensitivity.

\footnotetext{
*Corresponding author: e-mail: meilian201@163.com

** Corresponding author: e-mail: cfyang@nuk.edu.tw https://doi.org/10.18494/SAM.2021.3390
} 
Resistance distance is a distance function on a graph introduced by Klein and Randic, which is based on the theory of electrical networks. They found that a graphical distance can be used to indicate the effective resistance between pairs of vertices. ${ }^{(4,5)}$ In recent years, resistance distance has been widely used in random walk, electronic engineering, complex network, and chemical graph theory, and is attracting the attention of many scholars worldwide. ${ }^{(6-10)}$ The effective resistance between two nodes in an electrical network corresponds to the resistance distance $r\left(v_{i}, v_{j}\right)$ between two vertices of a graph. Let $\mathrm{G}$ be a graph with vertex set $\left\{v_{1}, v_{2}, \ldots, v_{n}\right\}$. The corresponding electrical network is obtained by replacing each edge of the graph with a fixed resistance (unit resistance). In the past, many researchers used the mathematical literature to study and calculate the resistance distance. Also, many studies were carried out to compute Kirchhoff indexes of specific classes of graphs, or to find bounds for Kirchhoff indexes of graphs and to characterize extremal graphs. For example, bicyclic and unicyclic graphs with extremal Kirchhoff indexes are characterized to obtain sharp bounds for Kirchhoff indexes of specific graphs. Throughout this study, we used a graph to model the molecular structure, in which each edge denotes a chemical bond and each vertex expresses an atom between two atoms.

\section{Exact Definition of Multiplicative Degree-Kirchhoff Index}

The multiplicative degree-Kirchhoff index of a connected graph is defined as the sum of the product of the resistance distances between all pairs of points and the degrees of corresponding point pairs in the graph. ${ }^{(7)}$

$$
K f^{*}(G)=\sum_{1 \leq i<j \leq n} d_{i} d_{j} r\left(v_{i}, v_{j}\right)=\frac{1}{2} \sum_{i=1}^{n} \sum_{j=1}^{n} d_{i} d_{j} r\left(v_{i}, v_{j}\right)=\frac{1}{2} \sum_{i=1}^{n} R_{D^{*}}\left(v_{i} \mid G\right)
$$

Here, $R_{D^{*}}\left(v_{i} \mid G\right)=\sum_{j=1}^{n} d_{i} d_{j} r\left(v_{i}, v_{j}\right)$. In this definition, $r\left(v_{i}, v_{j}\right)=0$.

The multiplicative degree-Kirchhoff index is an important topological index (invariant) of a graph, which is generated from the molecular structure and is a way of numerizing the molecular structure. It also reflects the structural characteristics of the compound. Polyphenyls are a type of macrocyclic aromatic hydrocarbon, which have attracted the attention of many chemists. ${ }^{(11-13)}$ Polyphenyl molecular graphs (or more precisely, graphs representing the carbon atoms) are called polyphenyl systems. If each vertex of a polyphenyl system lies in a hexagon and the graph obtained by contracting every hexagon to a vertex in the polyphenyl system is a path, we call this system a polyphenyl chain. Figure 1 shows the unique polyphenyl chains for $n=1,2$ and all the polyphenyl chains for $n=3,4$.

More generally, a polyphenyl chain $P P C_{n}$ with $n$ hexagons is regarded as a polyphenyl chain with $n-1$ hexagons by adding a cutting edge to a new hexagon, as shown in Fig. 2.(1)

For $n \geq 3$, the terminal hexagon can be attached in three ways, and the three local arrangements are denoted as $P P C_{n+1}^{1}, P P C_{n+2}^{1}$, and $P P C_{n+3}^{1}$, as shown in Fig. 3. ${ }^{(1)}$

A random polyphenyl chain $\operatorname{PPC}\left(n, p_{1}, p_{2}\right)$ with $n$ hexagons is obtained by the stepwise addition of terminal hexagons. At each step $(k=3,4, \ldots, n)$, a random selection is made from one 


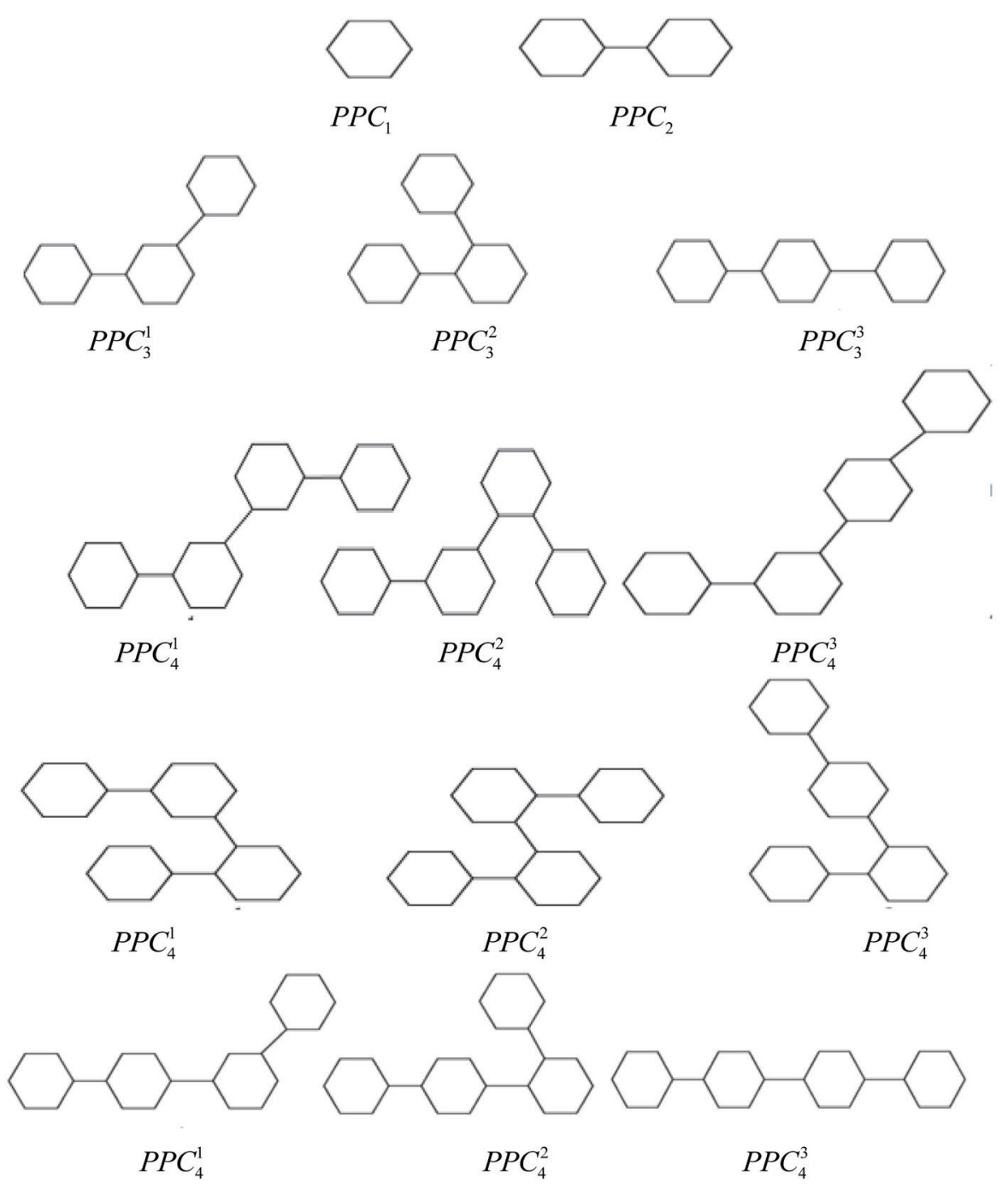

Fig. 1. Polyphenyl chains.

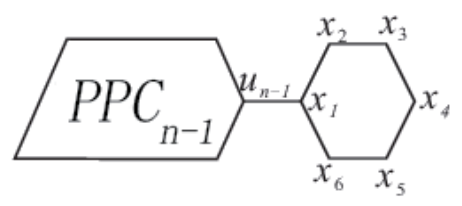

Fig. 2. Polyphenyl chain $P P C_{n}$ with $n$ hexagons.

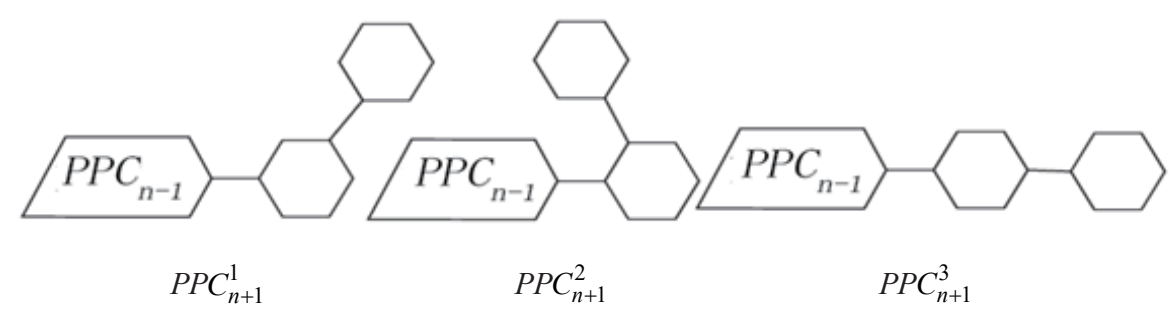

Fig. 3. Three local arrangements of polyphenyl chains. 
of the three possible constructions: (1) $P P C_{k-1} \rightarrow P P C_{k}^{1}$ with probability $p_{1}$, (2) $P P C_{k-1} \rightarrow P P C_{k}^{2}$ with probability $p_{2}$, and (3) $P P C_{k-1} \rightarrow P P C_{k}^{3}$ with probability $p_{3}=1-p_{1}-p_{2}$. The important novelty of this study is we assume that probabilities $p_{1}$ and $p_{2}$ are constants and invariant with the step parameter $k$. For a random polyphenyl chain $\operatorname{PPC}\left(n, p_{1}, p_{2}\right)$, the multiplicative degreeKirchhoff index $K f^{*}\left(\operatorname{PPC}\left(n, p_{1}, p_{2}\right)\right)$ is a random variable. Therefore, we propose an exact formula for its expected value $E\left(K f^{*}\left(P P C\left(n, p_{1}, p_{2}\right)\right)\right)$.

\section{Main Result}

Theorem 1: For $n \geq 1$, we have

$$
\begin{aligned}
E\left(K f^{*}\left(P P C\left(n, p_{1}, p_{2}\right)\right)\right) & =81 \frac{2}{3}-13\left(29 p_{1}+116 p_{2}-406\right)\left[1-\left(\frac{2}{3}\right)^{n-1}\right] \\
+ & {\left[\frac{13}{3}\left(105-7 p_{1}-28 p_{2}\right)+\frac{518}{3}\right]\left(n^{2}+n-2\right) } \\
+ & (n-1)\left[\frac{13}{3}\left(57 p_{1}+228 p_{2}-826\right)-\frac{484}{3}\right] .
\end{aligned}
$$

Proof: As described above, the polyphenyl chain $P P C_{n}$ is obtained by attaching $P P C_{n-1}$ to a new terminal hexagon by a cutting edge, as shown in Fig. 2. If the terminal hexagon is spanned by vertices $x_{1}, x_{2}, \ldots$, and $x_{6}$, then the new edge is denoted as $u_{n-1} x_{1}$, as shown in Fig. 2 . Note that

1.

$$
\begin{aligned}
& d_{1} d_{2} r\left(x_{1}, x_{2}\right)=3 \cdot 2 \cdot \frac{5}{6}=5, d_{1} d_{3} r\left(x_{1}, x_{3}\right)=3 \cdot 2 \cdot \frac{4}{3}=8, d_{1} d_{4} r\left(x_{1}, x_{4}\right)=3 \cdot 2 \cdot \frac{3}{2}=9 \\
& d_{1} d_{5} r\left(x_{1}, x_{5}\right)=3 \cdot 2 \cdot \frac{4}{3}=8, d_{1} d_{6} r\left(x_{1}, x_{6}\right)=3 \cdot 2 \cdot \frac{5}{6}=5
\end{aligned}
$$

Thus, we have $\sum_{j=1}^{6} d_{1} d_{j} r\left(x_{1}, x_{j}\right)=35$.

$$
\begin{aligned}
& \sum_{j=1}^{6} d_{1} d_{j} r\left(x_{1}, x_{j}\right)=35, d_{2} d_{1} r\left(x_{2}, x_{1}\right)=2 \cdot 3 \cdot \frac{5}{6}=5, d_{2} d_{3} r\left(x_{1}, x_{3}\right)=2 \cdot 2 \cdot \frac{5}{6}=\frac{10}{3}, \\
& d_{2} d_{4} r\left(x_{2}, x_{4}\right)=2 \cdot 2 \cdot \frac{4}{3}=\frac{16}{3}, d_{2} d_{5} r\left(x_{2}, x_{5}\right)=2 \cdot 2 \cdot \frac{3}{2}=6, d_{2} d_{6} r\left(x_{2}, x_{6}\right)=2 \cdot 2 \cdot \frac{4}{3}=\frac{16}{3} .
\end{aligned}
$$

So we have $\sum_{j=1}^{6} d_{2} d_{j} r\left(x_{2}, x_{j}\right)=25$.

$$
\begin{aligned}
& \sum_{j=1}^{6} d_{2} d_{j} r\left(x_{2}, x_{j}\right)=25, d_{3} d_{1} r\left(x_{3}, x_{1}\right)=2 \cdot 3 \cdot \frac{4}{3}=8, d_{3} d_{2} r\left(x_{3}, x_{2}\right)=2 \cdot 2 \cdot \frac{5}{6}=\frac{10}{3} \\
& d_{3} d_{4} r\left(x_{3}, x_{4}\right)=2 \cdot 2 \cdot \frac{5}{6}=\frac{10}{3}, d_{3} d_{5} r\left(x_{3}, x_{5}\right)=2 \cdot 2 \cdot \frac{4}{3}=\frac{16}{3}, d_{3} d_{6} r\left(x_{3}, x_{6}\right)=2 \cdot 2 \cdot \frac{3}{2}=6 .
\end{aligned}
$$


Thus, we have $\sum_{j=1}^{6} d_{3} d_{j} r\left(x_{3}, x_{j}\right)=26$.

$$
\begin{aligned}
& \sum_{j=1}^{6} d_{3} d_{j} r\left(x_{2}, x_{j}\right)=26, d_{4} d_{1} r\left(x_{4}, x_{1}\right)=2 \cdot 3 \cdot \frac{3}{2}=9, d_{4} d_{2} r\left(x_{4}, x_{2}\right)=2 \cdot 2 \cdot \frac{4}{3}=\frac{16}{3}, \\
& d_{4} d_{3} r\left(x_{4}, x_{3}\right)=2 \cdot 2 \cdot \frac{5}{6}=\frac{10}{3}, d_{4} d_{5} r\left(x_{4}, x_{5}\right)=2 \cdot 2 \cdot \frac{5}{6}=\frac{10}{3}, d_{4} d_{6} r\left(x_{4}, x_{6}\right)=2 \cdot 2 \cdot \frac{4}{3}=\frac{16}{3} .
\end{aligned}
$$

Also, we have $\sum_{j=1}^{6} d_{4} d_{j} r\left(x_{4}, x_{j}\right)=9+\frac{52}{3}$.

By symmetry, we have

$$
\sum_{j=1}^{6} d_{5} d_{j} r\left(x_{5}, x_{j}\right)=\sum_{j=1}^{6} d_{3} d_{j} r\left(x_{3}, x_{j}\right)=26, \sum_{j=1}^{6} d_{6} d_{j} r\left(x_{6}, x_{j}\right)=\sum_{j=1}^{6} d_{2} d_{j} r\left(x_{2}, x_{j}\right)=25
$$

Thus, we obtain $\sum_{i=1}^{6} \sum_{j=1}^{6} d_{i} d_{j} r\left(x_{i}, x_{j}\right)=35+25+26+9+\frac{52}{3}+26+25=146+\frac{52}{3}$.

2. $P P C_{n-1}$ has $6(n-1)$ vertices.

3. $\forall v \in P P C_{n-1}$,

$$
\begin{aligned}
& d_{1} d_{v} r\left(x_{1}, v\right)=3 d_{v}\left[r\left(u_{n-1}, v\right)+1\right], d_{2} d_{v} r\left(x_{2}, v\right)=2 d_{v}\left[r\left(u_{n-1}, v\right)+1+\frac{5}{6}\right], \\
& d_{3} d_{v} r\left(x_{3}, v\right)=2 d_{v}\left[r\left(u_{n-1}, v\right)+1+\frac{4}{3}\right], d_{4} d_{v} r\left(x_{4}, v\right)=2 d_{v}\left[r\left(u_{n-1}, v\right)+1+\frac{3}{2}\right], \\
& d_{5} d_{v} r\left(x_{5}, v\right)=2 d_{v}\left[r\left(u_{n-1}, v\right)+1+\frac{4}{3}\right], d_{6} d_{v} r\left(x_{6}, v\right)=2 d_{v}\left[r\left(u_{n-1}, v\right)+1+\frac{5}{6}\right] .
\end{aligned}
$$

The product of degree $x_{1}$ in $P P C_{n}$ is denoted by $R_{D^{*}}\left(x_{1} \mid P P C_{n}\right)$, and we have

$$
\begin{aligned}
R_{D^{*}}\left(x_{1} \mid P P C_{n}\right)= & \sum_{j \in P P C_{n}} d_{1} d_{j} r\left(x_{1}, x_{j}\right) \\
= & \sum_{v \in P P C_{n-1}} d_{1} d_{v} r\left(x_{1}, v\right)+d_{1} d_{2} r\left(x_{1}, x_{2}\right)+d_{1} d_{3} r\left(x_{1}, x_{3}\right) \\
& +d_{1} d_{4} r\left(x_{1}, x_{4}\right)+d_{1} d_{5} r\left(x_{1}, x_{5}\right)+d_{1} d_{6} r\left(x_{1}, x_{6}\right) \\
= & \sum_{v \in P P C_{n-1}} 3 d_{v}\left[r\left(u_{n-1}, v\right)+1\right]+35 .
\end{aligned}
$$

Similarly,

$$
R_{D^{*}}\left(x_{2} \mid P P C_{n}\right)=\sum_{v \in P P C_{n-1}} 2 d_{v}\left[r\left(u_{n-1}, v\right)+\frac{11}{6}\right]+25
$$




$$
\begin{gathered}
R_{D^{*}}\left(x_{3} \mid P P C_{n}\right)=\sum_{v \in P P C_{n-1}} 2 d_{v}\left[r\left(u_{n-1}, v\right)+\frac{7}{3}\right]+26, \\
R_{D^{*}}\left(x_{4} \mid P P C_{n}\right)=\sum_{v \in P P C_{n-1}} 2 d_{v}\left[r\left(u_{n-1}, v\right)+\frac{5}{2}\right]+9+\frac{52}{3}, \\
R_{D^{*}}\left(x_{5} \mid P P C_{n}\right)=\sum_{v \in P P C_{n-1}} 2 d_{v}\left[r\left(u_{n-1}, v\right)+\frac{7}{3}\right]+26, \\
R_{D^{*}}\left(x_{6} \mid P P C_{n}\right)=\sum_{v \in P P C_{n-1}} 2 d_{v}\left[r\left(u_{n-1}, v\right)+\frac{11}{6}\right]+25 .
\end{gathered}
$$

Also,

$$
\begin{aligned}
K f^{*}\left(P P C_{n}\right)= & K f^{*}\left(P P C_{n-1}\right)+R_{D^{*}}\left(x_{1} \mid P P C_{n}\right)+R_{D^{*}}\left(x_{2} \mid P P C_{n}\right)+R_{D^{*}}\left(x_{3} \mid P P C_{n}\right) \\
& +R_{D^{*}}\left(x_{4} \mid P P C_{n}\right)+R_{D^{*}}\left(x_{5} \mid P P C_{n}\right)+R_{D^{*}}\left(x_{6} \mid P P C_{n}\right)-\frac{1}{2} \sum_{i=1}^{6} \sum_{j=1}^{6} d_{i} d_{j} r\left(x_{i}, x_{j}\right) \\
& =K f^{*}\left(P P C_{n-1}\right)+13 \sum_{v \in P P C_{n-1}} d_{v} r\left(u_{n-1}, v\right)+\frac{74}{3} \sum_{v \in P P C_{n-1}} d_{v}+73+\frac{52}{6} \\
& =K f^{*}\left(P P C_{n-1}\right)+\frac{13}{3} \sum_{v \in P P C_{n-1}} d_{u_{n-1}} d_{v} r\left(u_{n-1}, v\right)+\frac{74}{3} \sum_{v \in P P C_{n-1}} d_{v}+73+\frac{52}{6} \\
& =K f^{*}\left(P P C_{n-1}\right)+\frac{13}{3} R_{D^{*}}\left(u_{n-1} \mid P P C_{n-1}\right)+\frac{74}{3}(14 n-16)+73+\frac{52}{6} .
\end{aligned}
$$

Then,

$$
K f^{*}\left(P P C_{n+1}\right)=K f^{*}\left(P P C_{n}\right)+\frac{13}{3} R_{D^{*}}\left(u_{n} \mid P P C_{n}\right)+\frac{74}{3} \cdot(14 n-2)+73+\frac{52}{6} .
$$

For a random polyphenyl chain $\operatorname{PPC}\left(n, p_{1}, p_{2}\right), R_{D^{*}}\left(u_{n} \mid P P C\left(n, p_{1}, p_{2}\right)\right.$ is a random variable, and we denote its expected value by $U_{n}=E\left(R_{D^{*}}\left(u_{n} \mid P P C\left(n, p_{1}, p_{2}\right)\right)\right)$. There are three cases to consider:

Case 1. $P P C_{n} \rightarrow P P C_{n+1}^{1}$. In this case, $u_{n}$ coincides with the vertex labeled $x_{3}$ or $x_{5}$. Consequently, $R_{D^{*}}\left(u_{n} \mid P P C\left(n, p_{1}, p_{2}\right)\right.$ is given by Eq. (11) or (13).

Case 2. $P P C_{n} \rightarrow P P C_{n+1}^{2}$. In this case, $u_{n}$ coincides with the vertex labeled $x_{2}$ or $x_{6}$. Consequently, $R_{D^{*}}\left(u_{n} \mid P P C\left(n, p_{1}, p_{2}\right)\right.$ is given by Eq. (10) or (14).

Case 3. $P P C_{n} \rightarrow P P C_{n+1}^{3}$. In this case, $u_{n}$ coincides with the vertex labeled $x_{4}$. Consequently, $R_{D^{*}}\left(u_{n} \mid P P C\left(n, p_{1}, p_{2}\right)\right.$ is given by Eq. (12).

Since the above three cases occur in random polyphenyl chains with probabilities $p_{1}, p_{2}$, and $1-p_{1}-p_{2}$, respectively, we immediately obtain the following result: 


$$
\begin{aligned}
U_{n} & =E\left(R_{D^{*}}\left(u_{n} \mid P P C\left(n, p_{1}, p_{2}\right)\right)\right) \\
& =p_{1}\left\{\sum_{v \in P P C_{n-1}} 2 d_{v}\left[r\left(u_{n-1}, v\right)+\frac{7}{3}\right]+26\right\}+p_{2}\left\{\sum_{v \in P P C_{n-1}} 2 d_{v}\left[r\left(u_{n-1}, v\right)+\frac{11}{6}\right]+25\right\} \\
& +\left(1-p_{1}-p_{2}\right)\left\{\sum_{v \in P P C_{n-1}} 2 d_{v}\left[r\left(u_{n-1}, v\right)+\frac{5}{2}\right]+9+\frac{52}{3}\right\} \\
& =p_{1}\left[\frac{2}{3} \sum_{v \in P P C_{n-1}} 3 d_{v} r\left(u_{n-1}, v\right)+\frac{14}{3} \sum_{v \in P P C_{n-1}} d_{v}+26\right] \\
& +p_{2}\left[\frac{2}{3} \sum_{v \in P P C_{n-1}} 3 d_{v} r\left(u_{n-1}, v\right)+\frac{11}{3} \sum_{v \in P P C_{n-1}} d_{v}+25\right] \\
& +\left(1-p_{1}-p_{2}\right)\left[\frac{2}{3} \sum_{v \in P P C_{n-1}} 3 d_{v} r\left(u_{n-1}, v\right)+5 \sum_{v \in P P C_{n-1}} d_{v}+9+\frac{52}{3}\right] \\
& =p_{1}\left[\frac{2}{3} R_{D^{*}}\left(u_{n-1} \mid P P C_{n-1}\right)+\frac{14}{3} \cdot(14 n-16)+26\right] \\
& +p_{2}\left[\frac{2}{3} R_{D^{*}}\left(u_{n-1} \mid P P C_{n-1}\right)+\frac{11}{3} \cdot(14 n-16)+25\right] \\
& +\left(1-p_{1}-p_{2}\right)\left[\frac{2}{3} R_{D^{*}}\left(u_{n-1} \mid P P C_{n-1}\right)+5 \cdot(14 n-16)+9+\frac{52}{3}\right] .
\end{aligned}
$$

By applying the properties of mathematical expectation to the above equation and noting that $E\left(U_{n}\right)=U_{n}$, we obtain

$$
\begin{aligned}
U_{n}= & p_{1}\left[\frac{2}{3} U_{n-1}+\frac{14}{3} \cdot(14 n-16)+26\right]+p_{2}\left[\frac{2}{3} U_{n-1}+\frac{11}{3} \cdot(14 n-16)+25\right] \\
& +\left(1-p_{1}-p_{2}\right)\left[\frac{2}{3} U_{n-1}+5 \cdot(14 n-16)+9+\frac{52}{3}\right] .
\end{aligned}
$$

This equation is easily transformed into

$$
U_{n}=\frac{2}{3} U_{n-1}+\left(70-\frac{14}{3} p_{1}-\frac{56}{3} p_{2}\right) n+5 p_{1}+20 p_{2}-\frac{161}{3} .
$$

The boundary condition is

$$
U_{1}=E\left(R_{D^{*}}\left(u_{1} \mid P P C\left(1, p_{1}, p_{2}\right)\right)=\sum_{j=1}^{6} d_{1} d_{j} r\left(x_{1}, x_{j}\right)=35 .\right.
$$


Using the above recurrence relation and the boundary condition, we obtain the value of $U_{n}$. Denoting $a=70-\frac{14}{3} p_{1}-\frac{56}{3} p_{2}, b=5 p_{1}+20 p_{2}-\frac{161}{3}$, we have

$$
U_{n}=\frac{2}{3} U_{n-1}+a \cdot n+b
$$

Denoting $d_{1}=U_{2}-U_{1}, d_{2}=U_{3}-U_{2}, \ldots, d_{n-1}=U_{n}-U_{n-1}$, we have

$$
\begin{aligned}
& U_{n}=d_{1}+d_{2}+\cdots+d_{n-1}+U_{1}, \\
& U_{n-1}=\frac{2}{3} U_{n-2}+a \cdot(n-1)+b .
\end{aligned}
$$

By subtracting Eq. (22) from Eq. (21), we obtain

$$
d_{n-1}=\frac{2}{3} d_{n-2}+a
$$

When $d_{1}=U_{2}-U_{1}=\frac{2}{3} \cdot 35+2 a+b-35=2 a+b-\frac{35}{3}$, from Eq. (23), we obtain

$$
\begin{aligned}
& d_{2}=\frac{2}{3} d_{1}+a, d_{3}=\frac{2}{3} d_{2}+a=\frac{2}{3}\left(\frac{2}{3} d_{1}+a\right)+a=\left(\frac{2}{3}\right)^{2} d_{1}+\left(1+\frac{2}{3}\right) a, \\
& d_{4}=\left(\frac{2}{3}\right)^{3} d_{1}+\left[1+\left(\frac{2}{3}\right)+\left(\frac{2}{3}\right)^{2}\right] a, \ldots, \\
& d_{n-1}=\left(\frac{2}{3}\right)^{n-2} d_{1}+\left[1+\left(\frac{2}{3}\right)+\left(\frac{2}{3}\right)^{2}+\cdots+\left(\frac{2}{3}\right)^{n-3}\right] a=\left(\frac{2}{3}\right)^{n-2} d_{1}+3 a \cdot\left[1-\left(\frac{2}{3}\right)^{n-2}\right]
\end{aligned}
$$

Then,

$$
\begin{aligned}
U_{n} & =d_{1}+d_{2}+\cdots+d_{n-1}+U_{1} \\
& =35+\left[1+\frac{2}{3}+\left(\frac{2}{3}\right)^{2}+\cdots+\left(\frac{2}{3}\right)^{n-2}\right] d_{1}+3 a \cdot\left\{\left(1-\frac{2}{3}\right)+\left[1-\left(\frac{2}{3}\right)^{2}\right]+\ldots+\left[1-\left(\frac{2}{3}\right)^{n-2}\right]\right\} \\
& =35+3 \cdot\left[1-\left(\frac{2}{3}\right)^{n-1}\right] d_{1}+3 a\left\{n-2-\left[\frac{2}{3}+\left(\frac{2}{3}\right)^{2}+\cdots+\left(\frac{2}{3}\right)^{n-2}\right]\right\} \\
& =35+3 \cdot\left[1-\left(\frac{2}{3}\right)^{n-1}\right] \cdot\left(2 a+b-\frac{35}{3}\right)+3 a\left\{(n-1)-3\left[1-\left(\frac{2}{3}\right)^{n-1}\right]\right\} \\
& =35+\left[1-\left(\frac{2}{3}\right)^{n-1}\right] \cdot(-3 a+3 b-35)+3 a(n-1) .
\end{aligned}
$$


Noting that $a=70-\frac{14}{3} p_{1}-\frac{56}{3} p_{2}, b=5 p_{1}+20 p_{2}-\frac{161}{3}$, from Eq. (25), we obtain

$$
U_{n}=35+\left(29 p_{1}+116 p_{2}-406\right)\left[1-\left(\frac{2}{3}\right)^{n-1}\right]+\left(210-14 p_{1}-56 p_{2}\right)(n-1) \text {. }
$$

Using the recurrence relation in Eq. (16), Eq. (26), and the expectation operator, we obtain

$$
\begin{aligned}
E\left(K f^{*}\left(P P C\left(n, p_{1}, p_{2}\right)\right)\right)= & E\left(K f^{*}\left(P P C\left(n-1, p_{1}, p_{2}\right)\right)\right)+\frac{13}{3} U_{n-1}+\frac{74}{3}(14 n-16)+73+\frac{52}{6} \\
= & E\left(K f^{*}\left(P P C\left(n-1, p_{1}, p_{2}\right)\right)\right)+\frac{74}{3}(14 n-16)+73+\frac{52}{6} \\
& +\frac{13}{3}\left\{35+\left(29 p_{1}+116 p_{2}-406\right)\left[1-\left(\frac{2}{3}\right)^{n-2}\right]+\left(210-14 p_{1}-56 p_{2}\right)(n-2)\right\} \\
= & E\left(K f^{*}\left(P P C\left(n-1, p_{1}, p_{2}\right)\right)\right)-\frac{13}{3}\left(29 p_{1}+116 p_{2}-406\right) \cdot\left(\frac{2}{3}\right)^{n-2} \\
& +\left[\frac{13}{3}\left(210-14 p_{1}-56 p_{2}\right)+\frac{1036}{3}\right] n+\frac{13}{3}\left(57 p_{1}+228 p_{2}-826\right)-\frac{484}{3} .
\end{aligned}
$$

The boundary condition is $E\left(K f^{*}\left(P P C\left(1, p_{1}, p_{2}\right)\right)\right)=73+\frac{52}{6}=81 \frac{2}{3}$, and using the above recurrence relation and the boundary condition, we have

$$
\begin{aligned}
E\left(K f^{*}\left(P P C\left(n, p_{1}, p_{2}\right)\right)\right)= & 81 \frac{2}{3}-13\left(29 p_{1}+116 p_{2}-406\right)\left[1-\left(\frac{2}{3}\right)^{n-1}\right] \\
& +\left[\frac{13}{3}\left(210-14 p_{1}+-56 p_{2}\right)+\frac{1036}{3}\right] \frac{n^{2}+n-2}{2} \\
& +(n-1)\left[\frac{13}{3}\left(57 p_{1}+228 p_{2}-\frac{484}{3}\right)\right] \\
& =81 \frac{2}{3}-13\left(29 p_{1}+116 p_{2}-406\right)\left[1-\left(\frac{2}{3}\right)^{n-1}\right] \\
& +\left[\frac{13}{3}\left(105-7 p_{1}-28 p_{2}\right)+\frac{518}{3}\right]\left(n^{2}+n-2\right) \\
& +(n-1)\left[\frac{13}{3}\left(57 p_{1}+228 p_{2}-826\right)-\frac{484}{3}\right]
\end{aligned}
$$

Finally, we point out the asymptotic property of $E\left(K f^{*}\left(P P C\left(n, p_{1}, p_{2}\right)\right)\right)$, which is

$$
E\left(K f^{*}\left(P P C\left(n, p_{1}, p_{2}\right)\right)\right) \sim\left[\frac{13}{3}\left(105-7 p_{1}-28 p_{2}\right)+\frac{518}{3}\right] n^{2}(n \rightarrow \infty) .
$$




\section{Conclusions}

The Kirchhoff index is not only an important invariant number characterizing bicyclic and unicyclic graphs, but is also an important means of describing molecular structures in chemistry. The Kirchhoff index also has important applications in the quantitative structure-activity relationships and quantitative structure-property relationships. It is very difficult to compute the Kirchhoff index from the computational complexity of the graph, and it is also difficult to obtain a specific formula for calculating the multiplicative degree-Kirchhoff index of a general graph. Therefore, the problem of finding the Kirchhoff index for some special graphs has attracted the widespread attention of researchers. In this study, we used the digital features of random variables and the methods and technologies of algebra to obtain the multiplicative degreeKirchhoff index of a random polyphenyl chain. We also consider that the development potential nature of the Kirchhoff index in physics, chemistry, and other fields will have further applications.

\section{Acknowledgments}

This work was supported by project numbers NSFC 11601214, JAT 160492, MOST 108-2221E-390-005, and MOST 109-2221-E-390-023.

\section{References}

1 G. Huang, M. Kuang, and H. Deng: Ars Math. Contemp. 9 (2015) 197.

H. Bai and G. Shi: Sensors 7 (2007) 267.

T. Graunke, K. Schmitt, and J. Wollenstein: J. Sens. 2016 (2016) 2435945.

4 S. Li, L. Shi, and W. Gao: Int. J. Quantum Chem. 121 (2021) e26589.

5 D. J. Klein and M. Randic: J. Math. Chem. 12 (1993) 81.

6 W. Yang and F. Zhang: Match Commun. Math. Comput. Chem. 68 (2012) 371.

7 H. Chen and F. Zhang: Discr. Appl. Math. 155 (2007) 654.

8 A. D. Mednykh and I. Mednykh: Doklady Math. 102 (2021) 392.

9 S. Huang and S. Li: Phys. A Statis. Mech. Applic. 558 (2020) 124999.

10 M. S. Sardar, X. F. Pan, and S. A. Xu: Appl. Math. Comput. 381 (2020) 125283.

11 D. R. Flower: J. Chem. Inf. Comput. Sci. 38 (1998) 379.

12 Q. R. Li, Q. Yang, H. Yin, and S. Yang: J. Univ. Sci. Technlo. Chain 34 (2004) 335.

13 S. Tepavcevic, A. T. Wroble, M. Bissen, D. J. Wallace, Y. Choi, and L. Han-ley: J. Phys. Chem. B 109 (2005) 7143. 